\section{hommes
\& migrations}

\section{Hommes \& migrations}

Revue française de référence sur les dynamiques

migratoires

$1310 \mid 2015$

Fashion Mix

\title{
«Blédard » et « fashion victim »
}

Du bon usage de la mode chez des adolescents d'un quartier populaire

\section{Aurélia Mardon et Zaihia Zeroulou}

\section{(2) OpenEdition}

1 Journals

\section{Édition électronique}

URL : http://journals.openedition.org/hommesmigrations/3162

DOI : 10.4000/hommesmigrations.3162

ISSN : 2262-3353

Éditeur

Musée national de l'histoire de l'immigration

\section{Édition imprimée}

Date de publication : 1 avril 2015

Pagination : 101-108

ISBN : 978-2-919040-31-5

ISSN : 1142-852X

Référence électronique

Aurélia Mardon et Zaihia Zeroulou, « «Blédard » et «fashion victim» », Hommes \& migrations [En ligne], 1310 | 2015, mis en ligne le 01 avril 2018, consulté le 20 avril 2019. URL : http://

journals.openedition.org/hommesmigrations/3162 ; DOI : 10.4000/hommesmigrations.3162 


\title{
"BLÉDARD" \\ ET "FASHION VICTIM" \\ DU BON USAGE DE LA MODE CHEZ DES ADOLESCENTS D'UN QUARTIER POPULAIRE
}

\author{
Par AURÉLIA MARDON, sociologue, université Lille-I/Clersé (UMR CNRS 8019) \\ et ZAIHIA ZEROULOU, sociologue, université Lille-I/Clersé (UMR CNRS 8019).
}

\begin{abstract}
Au collège, l'habit semble faire le moine. La sociabilité très conformiste des adolescents les pousse à s'aligner sur les codes de leurs pairs. C'est ce que montre l'analyse du rapport que des élèves issus d'un quartier populaire entretiennent à l'égard de la mode. Car leur façon de se vêtir, et notamment le choix des marques, influence leur réputation au sein de leur établissement. L'étude du contrôle qu'exercent les parents et les protagonistes du monde scolaire met en évidence la façon dont les élèves négocient les normes émises par les adultes.
\end{abstract}

Les pratiques corporelles et vestimentaires occupent une place centrale dans la construction de l'identité sociale ${ }^{1}$. Le vêtement donne des informations sur les caractéristiques sociales et personnelles des individus et témoigne de leur adhésion à des normes collectives sans cesse renouvelées. C'est le cas des adolescents qui, à travers leur apparence, expriment leurs goûts culturels ${ }^{2}$, leur appartenance générationnelle, leur maturité et leur individualité3. Leurs pratiques plurielles varient en fonction du genre, de l'origine sociale, des pratiques éducatives parentales et du climat social de l'établissement scolaire ${ }^{4}$. Pour autant, quelle connaissance avonsnous des effets de la variable "origine immigrée" sur la façon dont les adolescents mettent en scène leur corps et leur apparence au début de l'adolescence? Connaissons-nous les pratiques de transmissions/ transgressions familiales qui accompagnent la socialisation vestimentaire juvénile ${ }^{5}$ ? Les travaux de David Lepoutre sur la culture des adolescents de la Cité des 4000 montrent que ces jeunes, en partie issus de familles immigrées, partagent une culture commune, qui doit plus aux industries culturelles et aux musiques populaires comme le rap et le R'n'B 
qu’à leurs parents ${ }^{6}$. Quelles sont les conséquences sur le plan du corps?

Cet article tente d'apporter des éléments de réponse en adoptant le prisme des pratiques vestimentaires $^{7}$ de collégiens scolarisés dans un établissement situé au cœur d'une cité HLM de la banlieue parisienne ${ }^{8}$ et fréquenté par des élèves d'origine populaire ${ }^{9}$ et moyenne, issus notamment de familles algériennes, marocaines ou maliennes. Il prend le parti de ne pas isoler a priori ces enfants d'immigrés des autres adolescents avec lesquels ils partagent des influences communes. En effet, socialisés en France, ils possèdent des caractéristiques de leur génération ${ }^{10}$, Les pratiques vestimentaires des collégiens des quartiers des grands ensembles

s'inscrivent dans une logique de conformisme à l'égard des codes en vigueur dans leur établissement. mais ils ne constituent pas une "catégorie homogène (...) avec des frontières clairement définies ${ }^{11}$. Un même processus, l'émigration des parents, opère diversement, quant au fond et à la forme, sur l'appropriation ou le rejet de codes vestimentaires "traditionnels", et l'adoption de nouveaux codes, sans exclure la création de nouvelles façons de s'habiller $^{12}$. Ces jeunes s'inscrivent dans des processus globaux de complexification culturelle ${ }^{13}$. Par ailleurs, en tant que membres des classes populaires, ils résident dans un quartier d'habitat social, sont soumis à des contraintes similaires et adhèrent aux mêmes références culturelles, d'où l'appellation "adolescents issus d'un quartier populaire" pour désigner la population enquêtée. En resituant les jeunes dans la sociabilité juvénile, le rôle des adultes, notamment des parents, dans leurs rapports à l'apparence est questionné. Si la période du collège constitue le moment de l'émancipation parentale, elle n'en reste pas moins contrôlée par ces derniers. Les pratiques de ces jeunes sont saisies dans leur univers scolaire et dans leurs interactions avec l'équipe pédagogique et administrative. L'article repose sur une enquête réalisée par observation dans les classes et la cour de récréation et par entretiens individuels et collectifs auprès de 26 collégiens et collégiennes âgés de 11 à 15 ans dans le “collège J" durant les années 2005 et $2006^{14}$. Non classé en zone d'éducation prioritaire, il bénéficie de médiateurs ${ }^{15}$ et possède une section d'enseignement général et professionnel adapté.

\section{Tenir son rang et construire sa réputation en milieu scolaire}

Les pratiques vestimentaires des collégiens des quartiers des grands ensembles s'inscrivent dans une logique de conformisme à l'égard des codes en vigueur dans leur établissement. Collège et lycée constituent des scènes sociales où les jeunes sont soumis aux regards et aux jugements de leurs pairs $^{16}$. Tout jeune assure son prestige et sa réputation en respectant les codes vestimentaires de sa génération valorisés dans son collège. Ceux et celles qui affichent une apparence trop inspirée des goûts parentaux sont stigmatisés, qualifiés de "vieux gars" ou de "blédards", notions qui renvoient à un même contenu et que les collégiens utilisent pour désigner ceux qui ne vivent pas dans leur temps générationnel. Si les élèves de $\sigma^{\text {e }}$ sont excusés, parce qu'ils sont "petits", les autres sont "charriés", comme cet élève de $5^{\mathrm{e}}$ à qui un de ses camarades mi-moqueur, mi-sérieux lance lors d'un entretien collectif : "Lui, il s'habille comme un blédard!" Cette appellation rappelle une appartenance

6. David Lepoutre, Cour de banlieue. Codes, rites et langages, Paris, Odile Jacob, 1997. 7. II se centre sur les usages du vêtement en incluant coiffure, bijoux, maquillage. 8. Le collège est situé dans la commune de M., qui compte 15 ooo habitants. Séparé du reste de la ville par la forêt domaniale, il est implanté au cœur d'un grand ensemble créé dans les années 1960 et rassemble 393 élèves. 9. Les données statistiques disponibles et établies par l'inspection académique sur la base des déclarations des parents en 2005 révèlent que $33,8 \%$ d'entre eux appartiennent aux couches "défavorisées" de la population, 37,7 \% sont classés dans la catégorie "moyenne", 13, 7 \% dans la catégorie “favorisée B", et 14,8\% dans la catégorie “favorisée A". 10. Zaihia Zéroulou, op. cit., p. 176. 11. Bernard Preel, Les Générations mutantes. Belle Époque, Krach, Mai 68, Internet. Quatre générations dans l'histoire, Paris, La Découverte, 2005, p. 272.12. Zaihia Zéroulou, op. cit., p. 176. 13. Alain Battegay, “Les cultures incertaines des jeunes issus de l'immigration maghrébine”, in Hommes \& Migrations, $\mathrm{n}^{\circ}$ 1231, 2001, pp. 5-14. 14. Ce travail a été réalisé dans le cadre d'une thèse de sociologie intitulée "La socialisation corporelle des préadolescentes", soutenue à l'université Paris Ouest-Nanterre-La Défense en 2006. Elle porte sur la socialisation corporelle des préadolescentes, appréhendée à travers deux de ses enjeux, les transformations corporelles durant l'adolescence et la socialisation vestimentaire. 
tant jeans ou pantalons baggy, associés à des tee-shirts ou à des sweats très larges. En affirmant un style vestimentaire, ces jeunes exhibent leur maturité. Les styles sont reliés à des goûts culturels et sportifs qui transmettent aux jeunes des consignes concernant leur langage, et leurs manières de se vêtir et d'être avec les autres ${ }^{17}$. Ils s'inscrivent dans une logique médiaticopublicitaire qui, tout en valorisant une variété de styles et l'idée d'une production autonome de l'image qu'on donne de soi, cache en fait une homogénéisation des goûts. Le style adopté de façon majoritaire par les garçons s'appuie sur les valeurs de la culture de la rue identifiées par David Lepoutre $^{18}$ : l'art de la vanne, la force, le courage physique et la nécessité d'acquérir les marques de vêtements Nike, TN, Adidas, Sergio Tachini, Puma pour tenir son rang et conserver la face ${ }^{19}$. Le marché, lieu d'acquisition pour plusieurs familles, n'est pas considéré comme légitime à leurs yeux, ce qu'explique Tony, 12 ans, en classe de $5^{\mathrm{e}}$ :

LAfrique de la mode au Palais de la Porte Dorée, novembre 2010. (c) Abdelkader Benamer / D. Langoutte

sociale défavorisée et vécue de façon honteuse et déshonorante, parce qu'elle empêche de tenir son rang et de garder la face en accédant aux vêtements de marque. À l'inverse, il est plutôt valorisant d'être considéré comme une "fashion victim". Succomber aux attraits de la société de consommation est perçu positivement par ces élèves d'origine populaire.

Dans ce collège, les pratiques vestimentaires s'avèrent relativement homogènes et largement influencées par des musiques populaires tels que le rap et le R'n'B. Nombre de garçons disent s'habiller "à la ricain" ou à "l'américaine", en por-
"Y en a qui charrient. Y disent: 'T'as acheté tes Nike au marché !' L'attachement aux marques est une manière discrète pour les garçons de revendiquer en public l'identification à des héros masculins, comme l'a démontré Dominique Pasquier ${ }^{20}$. L'importance accordée à la coiffure, largement inspirée du monde sportif et musical et à l'identification à des joueurs de football, de basket ou à des chanteurs de rap, relève d'une logique similaire. Si la plupart des garçons ont les cheveux courts, voire rasés à la tondeuse, soit sur la totalité du cuir chevelu, soit sur les côtés, à l'exception de quelques garçons de familles africaines dont les cheveux sont nattés ou tressés, c'est que pour eux les cheveux longs signifient un manque de virilité. Ces codes capillaires correspondent à une cer- 
taine définition de la masculinité. Un élève de $5^{\mathrm{e}}$ résume la position de tous lors d'une discussion dans la salle de permanence: "Les cheveux longs, ça fait pédale!" Les élèves partagent une conception traditionnelle de la masculinité qui s'oppose à celles mises en scène par les jeunes de milieux plus favorisés ${ }^{21}$. Certains portent des casquettes ou des capuches, quelques garçons possèdent un brillant à l'oreille plus gros que ceux portés par les femmes mais ne dépassant pas un
Ceux et celles qui souhaitent se distinguer en affichant des styles vestimentaires dévalorisés s'exposent aux moqueries, voire à des manœuvres physiques d'intimidation. centimètre, ils sont largement inspirés par le rap et le style des rappeurs. Aucun ne porte d'anneaux aux oreilles ni de piercing qui, comme pour les cheveux longs, renvoient, dans ce contexte, à une absence de virilité.

Les filles disent suivre la mode en adoptant les tendances valorisées dans les magasins populaires tels que Pimkie, H\&M ou Zara et s'identifier à des figures du monde de la musique rap et R'n'B. Elles adhèrent majoritairement aux codes dominants de la féminité. La mode est aux vêtements près du corps et aux pulls cintrés. Les plus âgées viennent au collège maquillées et portent des vêtements sexués, comme en témoigne le portrait de Kardiatou, 14 ans, qui, selon ses camarades, représente bien les tendances de la mode. Bonne élève, elle est dans la classe européenne du collège. Ses parents sont maliens, son père employé et sa mère femme au foyer, elle a deux frères et une grande sœur. Elle et ses amies citent le rap, le R'n'B, et le reggae comme leurs goûts musicaux préférés. L'apparence de cette jeune fille est savamment travaillée, ses cheveux sont nattés et relevés en chignon. Elle a parsemé ses nattes d'anneaux gravés de couleur argent, posé un faux piercing sur son nez et collé une pierre brillante sur l'une de ses incisives. Elle porte généralement des vêtements près du corps et fait usage de maquillage en ornant ses paupières de fard blanc, ses cils de mascara et ses lèvres de gloss.

\section{Des codes vestimentaires partagés par tous, ou presque}

Le prestige des collégiens se construit en lien avec le respect de ces codes vestimentaires. Les plus jeunes apprennent à abandonner leurs vêtements d'enfant en entrant au collège et à respecter les normes d'âge précises régissant les relations des collégiens. Le portrait de Zorha, 12 ans, en classe de $5^{\mathrm{e}}$, révèle la logique de l'affichage de la "juste maturité". Les parents de Zohra sont marocains, son père est employé municipal et sa mère aide ménagère. Sa grande sœur et son grand frère fréquentent le même collège. Zohra, bonne élève, a le souci de coller aux codes valorisés dans son collège, qu'elle décrypte avec l'aide de ses copines et de sa sœur qu'elle admire, tout en l'enviant : "Y a déjà plusieurs garçons qui se sont battus pour elle." Les pratiques de sa sœur lui indiquent qu'il faut suivre la mode en respectant les normes d'âge de l'affichage de la féminité. Ainsi, elle précise : "Y a les petites chaussures pointues avec les tout petits talons, ma sour en a et je trouve ça super joli, mais moi je peux pas les mettre... Elles vont dire que je m'habille trop grande." Pourtant, elle s'est sentie flattée lorsque, se rendant en classe avec une nouvelle coupe de cheveux, elle a fait l'objet de l'admiration de ses camarades: "J'avais mis une jupe blanche et des collants et j'avais ma nouvelle coupe et tout le monde me regardait comme si jétais je sais pas quoi..."

Ceux et celles qui souhaitent se distinguer en affichant des styles vestimentaires dévalorisés s'exposent aux moqueries, voire à des manœuvres physiques d'intimidation. C'est le cas de quelques filles exprimant leur goût pour le métal et le rock gothique. Ce style associé à l'usage de vêtements noirs ou de tee-shirts à l'effigie d'un groupe portés avec des pantalons larges exprime la volonté de se distinguer qui se lit dans le détournement des codes de l'esthétique féminine. Le maquillage doit être noir, les jupes accompagnées de chaussures 
empruntées au monde du travail ouvrier masculin les "Docs" ou militaire les "rangers", les collants à rayures ou déchirés, les bijoux en argent, métal, cuir ou plastique noir en forme de pics. Les garçons de la section d'enseignement général et professionnel adapté (Segpa) se mettent en valeur et construisent leur héroïsme en glosant sur leur attitude de défiance à l'égard de ces élèves. Ainsi, l'un d'entre eux, en entretien, valorise un de ses camarades présent en rappelant: "Ici, y a plus de gothiques, $X$ les a traumatisés.” À travers les antagonismes et les tensions suscités par les styles choisis, les collégiens apprennent que des affirmations stylistiques sont plus rentables pour le travail de sociabilité que d'autres, certaines s'avérant catastrophiques. Ce qui explique que des filles changent de style au cours de leur scolarité, comme Lisa, jeune gothique de 14 ans, qui tente désormais de témoigner de son goût pour la musique reggae, en coiffant ses cheveux blonds "à l'afro". Si aucun garçon n'affiche de goûts trop éloignés des codes majoritaires, c'est parce que la transgression des normes de genre est trop coûteuse pour ceux qui souhaitent bénéficier d'une visibilité sociale valorisante dans l'établissement.

Collégiens et collégiennes se conforment aux usages dominants et conservent également des marqueurs culturels pour venir à l'école. Des filles portent un collier avec leur prénom en arabe ou des bijoux achetés lors de voyages dans le pays d'origine des parents. Ces signes restent discrets, chacun se faisant un devoir d'afficher son adhésion aux normes de tous, comme en témoigne l'incident survenu un matin dans le bureau de la vie scolaire. En retard, un élève se présente pour prévenir les médiateurs en oubliant d'enlever la djellaba qu'il portait chez lui. Les surveillants, dont l'un est issu d'une famille algérienne, l'accueillent en se moquant de sa tenue, lui signifiant son incongruité dans le contexte scolaire. "Va me chercher le boutiquier", dit l'un d'eux, ce qui fait rire les adultes présents, le garçon comprenant qu'on se moque de lui ${ }^{22}$.
Comme nombre d'adolescents, ces collégiens, soumis au contrôle parental, sont amenés à négocier l'acquisition et l'usage de certains vêtements, développant parfois des pratiques en secret, des ruses pour accéder à leur autonomie.

\section{Avec les parents : entre appropriation, négociation et transgression}

Ces collégiens restent dépendants de leurs parents pour l'achat d'habits qui, comme dans de nombreuses familles populaires, constituent aussi une occasion de "faire plaisir" aux enfants ${ }^{23}$. Zorha, 12 ans, en classe de 5 e, explique la volonté paternelle de "gâter" ses enfants: "Il veut nous gâter parce qu'il nous a dit: 'Avant, j'ai souffert, jétais dans la misère.' Parce qu'il habitait au bled, pardon, au Maroc, il veut nous apporter tout le bonheur." Dans ces familles aux fratries importantes, l'achat de vêtements donne lieu à un paradoxe : tout en voulant faire plaisir, on incite à hiérarchiser les demandes, comme en témoignent les propos de Zorha : "Y a pas longtemps, on a acheté un pantalon à mon père, on m'a acheté aussi un parfum... et maintenant quand je demande des habits: 'Ah bah t'as déjà eu ton parfum..."

Même en restant tributaires des choix ou des contraintes Le contrôle des parents conduit les filles à ruser pour afficher les codes de leur génération en dissimulant le maquillage, en profitant du départ des parents avant l'école pour choisir leurs tenues et se changer dans l'ascenseur,

voire au coin de la rue. des parents, collégiens et collégiennes démontrent leur indépendance dans ce domaine en remettant en cause les goûts parentaux, comme Aïcha, 14 ans, en $4^{\mathrm{e}}$ : "Les parents, ils achètent des trucs moches!" ou encore Kardiatou, qui souligne: "Ma mère, elle cherche pas trop à comprendre mon monde!" La construction de cette indépendance est progressive, les pratiques corporelles témoignent des limites imposées par les parents. 


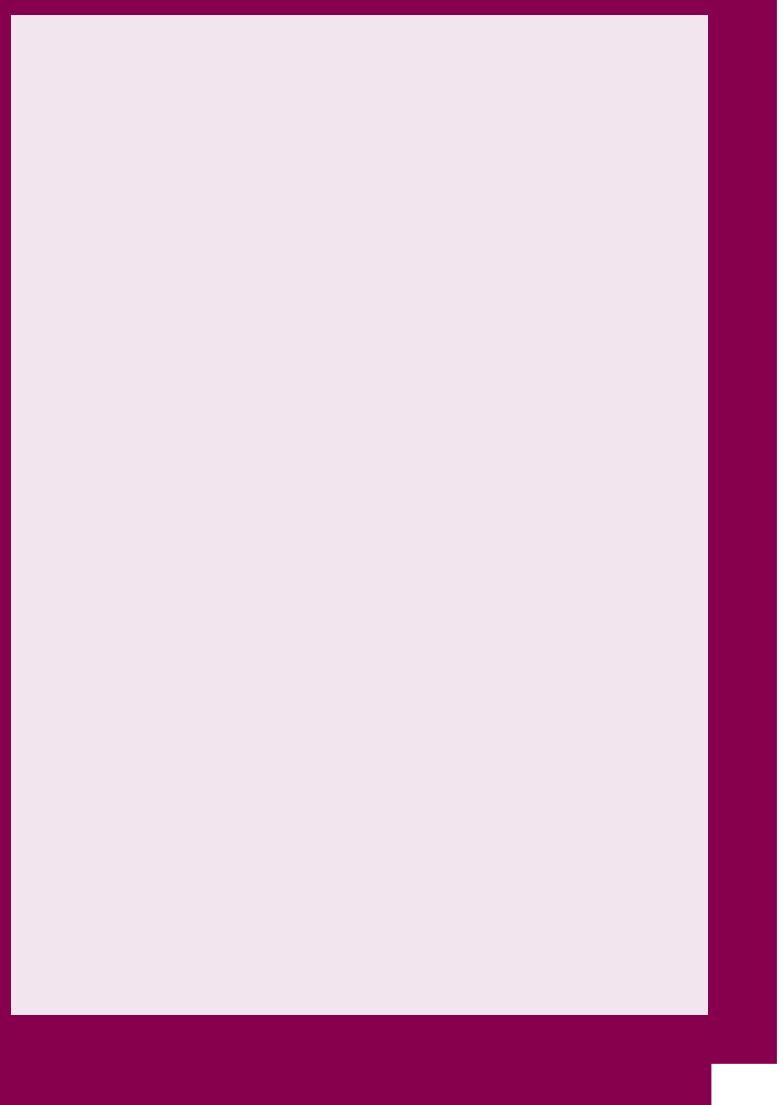

L'Afrique de la mode au Palais de la Porte Dorée, novembre 2010. (c) Abdelkader Benamer / D. Langoutte

C'est le cas pour Aïcha, dont la mère refuse qu'elle se fasse couper ses longs cheveux. Les parents édictent également des normes d'âge quant à la mise en valeur du corps pour les filles, sans pour autant l'interdire. Zorha évoque celles de sa mère : “Quand je dis à ma mère: 'Tu peux m'acheter ça?' Elle me dit: 'C'est un peu trop décolleté pour ton âge. Attends un peu plus tard."

Le contrôle des parents conduit les filles à ruser pour afficher les codes de leur génération en dissimulant le maquillage, en profitant du départ des parents avant l'école pour choisir leurs tenues et se changer dans l'ascenseur, voire au coin de la rue. Plus que les garçons, les filles témoignent de l'in- tériorisation des normes parentales, qui ne sont pas propres aux milieux populaires ou aux familles immigrées ${ }^{24}$, lorsque qu'elles déclarent faire preuve de retenue. Par exemple, Zohra souhaite ne pas passer pour quelqu'un qui "se montre", traduisant l'idée, très présente dans les entretiens, qu'une fille doit respecter des limites : pas de décolletés trop plongeants, de jupes trop courtes, pas de maquillage trop prononcé, pas de tenues "trop exagérées", "trop vulgaires" pour ne pas faire "pute". Les garçons contrôlent les pratiques des filles de manière indirecte par le biais de remarques, de regards ou de ragots. Pour décrire l'apparence féminine, ils utilisent un langage obscène ou grossier parce que celle-ci engage la sexualité et la moralité des femmes. La crudité verbale fait partie, comme l'a souligné David Lepoutre ${ }^{25}$, de la culture des rues, s'affirmant en opposition au langage des adultes, aux manières de parler bourgeoises et à la langue française pour les enfants d'immigrés. Parallèlement aux normes émises par les parents et intériorisées par les enfants, les protagonistes du monde scolaire participent également à la socialisation vestimentaire des collégiens.

\section{Avec les protagonistes du monde scolaire : entre soumission, indignation et résistance}

Parents et membres de l'équipe éducative acceptent que les élèves revendiquent leur individualité et leur authenticité à travers leur apparence. L'image d'une enfance ayant ses qualités propres et des besoins d'expression particuliers ${ }^{26}$ se substitue à celle d'une enfance incomplète et devant être éduquée. Les adultes ne remettent pas en cause les styles adoptés par les élèves et la légitimité d'exprimer leur singularité à travers leur façon de 
s'habiller. Toutefois, si l'apparence des publics scolaires fait l'objet de débats dans la presse à propos du respect de la laïcité, d'autres questions préoccupent l'équipe éducative, comme en témoignent plusieurs articles du règlement intérieur du collège dans la rubrique "Respect d'autrui et politesse". Le premier précise que "dans l'enceinte du collège, les élèves doivent... proscrire les vêtements négligés, déchirés et/ou provocants". Un deuxième exige que leurs manières de se vêtir ne fassent pas courir des risques physiques. Un troisième contraint les élèves à respecter les normes de la pudeur vestimentaire. Les élèves qui cultivent de leur propre aveu un "laisser-aller" transgressent les obligations. Les filles qui exhibent leur féminité en dévoilant et en sexualisant leur corps sont directement concernées. Nos observations montrent que si garçons et filles sont rappelés à l'ordre et réprimandés, les causes ne sont pas de même nature et elles ne font pas peser sur les uns et les autres les mêmes contraintes. Les garçons sont généralement interpellés pour leur look négligé, le port de pantalons baggy, qu'ils sont sommés de remonter. Ils peuvent aussi se voir rappeler certaines parades sexuées, comme en témoigne cette scène saisie lors d'une récréation. Un garçon de la section Segpa, ayant des cheveux bouclés et plus longs que la normale, est interpellé par un surveillant à l'heure du repas : "Quand est-ce que tu vas te couper les cheveux? Comment tu vas séduire ta future femme coiffé comme ça?"

Les filles faisant étalage de leur goût pour la musique métal ou gothique sont rappelées à l'ordre lorsqu'elles arborent des accessoires considérés comme transgressifs tels que les colliers ou les bracelets à pics. Mais les remarques adressées aux filles visent surtout à leur faire respecter les normes de la décence et celles de l'ajustement de leur tenue à leur âge ${ }^{27}$. Les protagonistes du monde scolaire vitupèrent contre la façon dont les filles associent pantalon taille basse et T-shirt découvrant leur nombril, le recours à des jupes "très courtes" ou a un maquillage "trop prononcê". Les enseignants de lycée imaginant avant tout leurs élèves au masculin $^{28}$, on comprend que les manifestations "précoces" de féminité des collégiennes agacent les professeurs et le personnel administratif. Elles introduisent de la féminité dans un espace censé en être dépourvu et trop tôt selon les normes des adultes. Claude Zaidman montre que les petites filles sont engagées à se comporter en fonction des réactions éventuelles des garçons ${ }^{29}$. Au collège, l'équipe éducative insiste sur le danger des tenues indécentes dans l'espace public, parce qu'elles attirent le regard des garçons. Les attitudes et comportements des hommes sont mis en cause, mais on incite les filles à adapter leurs attitudes vestimentaires aux regards masculins et à adopter les normes d'une féminité mesurée. L’observation présentée cidessous cristallise l'ensemble des griefs adressés aux filles dans létablissement et montre comment les surveillants participent à la transmission des
Quand on interroge les élèves sur les remarques que les adultes leur adressent à propos de leur apparence, l'irritation n'est jamais loin. normes de la bonne mesure.

Les "extravagances" vestimentaires de Nadia, en $3^{e}$, sont souvent discutées au cours de l'année. Un jour, lorsquelle entre dans le bureau de la vie scolaire, une surveillante commente sa tenue : "Regarde, elle l'a enlevé mais on voyait tout." Ce jourlà, elle est vêtue d'un jean large et taille basse porté avec une ceinture serrée, parce qu'un membre de l'équipe éducative l'a obligée à se couvrir. La surveillante surenchérit à propos de son maquillage : "T'as pas le visage qui est attiré vers le bas ?" Nadia trouve la réflexion déplacée et le manifeste avant de sortir brutalement du bureau en déclarant : "C'est bon, je suis pas venue ici pour me faire charrier!" Quelqu'un sort pour la ramener dans le bureau. Lorsque l'élève entre à nouveau, elle se fait sermonner: "Quand on te dit quelque chose, tu n'as pas à manifester ton mécontentement, c'est nous les adultes ici !" Elle est à nouveau accablée de remontrances : "Qu'est-ce qu'il y a, t'es pas fashion si on voit pas le bout 
de ton ventre ?" L'élève rétorque : "Non mais avec ce pantalon, ça va pas, j'ai l'air d'une clocharde." La surveillante reprend: "Regarde... t'as une peau de pêche, t'as pas besoin d'en mettre autant." L'élève reste silencieuse, sort du bureau avec moins de fracas. Plus tard, alors que nous nous entretenons avec elle au sujet de cette scène, elle fait part de son indignation: "Vous trouvez pas que c'est abuser ça ?" (bureau de la vie scolaire, 13 mars 2006).

\section{Un contrôle institutionnel de l'apparence}

Celles qui s'habillent comme des garçons choquent tout autant. Ainsi, une jeune fille en classe de $3^{e}$ fait l'objet de regards désapprobateurs d'un surveillant, au sujet de son apparence jugée trop masculine. L'école favorise ainsi l'image d'une apparence mesurée, féminine mais sans tomber dans l'excès.

De leur côté, les élèves considèrent que l'école abuse de ses prérogatives en faisant des critiques, remarques ou allusions qui empiètent sur leur vie privée. Amandine, 12 ans, raconte avec colère qu'une de ses amies a été réprimandée par la principale parce qu'elle était trop légèrement vêtue. Quand on interroge les élèves sur les remarques que les adultes leur adressent à propos de leur apparence, l'irritation n'est jamais loin. "Ils sont vraiment à cran sur la tenue vestimentaire ici", explique Justine, 13 ans, qui souligne qu'un "bout de nombril à l'air" provoque "une crise". Les filles régulièrement rappelées à l'ordre ne reconnaissent pas la légitimité de l'institution, elles remettent en cause les normes qu'elle veut leur imposer. Ainsi, des filles de $3^{\text {e }}$ soulignent que, contrairement à ce que pensent les adultes, découvrir ses épaules dans le cadre scolaire n'est pas "vulgaire", d'autres réfutent l'idée que le port de bracelets à pics puisse s'avérer "dangereux", puisque les pics en question sont "arrondis". Une autre se trouvant dans la cour du collège, et à qui un surveillant demande en notre présence pourquoi elles s'habillent toutes comme "des petites dames", répond: "J'ai bientôt 16 ans quand même!" Les élèves résistent au poids des injonctions des adultes et les détournent en utilisant diverses tactiques pour assurer leur intégrité vestimentaire, celles-ci traduisent une volonté d'accéder à d'autres places et rôles que ceux désignés par les adultes ${ }^{30}$.

Pour affiner ces résultats, il faudrait inclure les usages du vêtement dans l'espace privé et festif de ces jeunes et dans le pays d'origine des parents. On peut faire l'hypothèse que lorsqu'ils se retrouvent dans un contexte familial, où le milieu migratoire est dominant, ils ont plus souvent recours à des tenues vestimentaires valorisées au sein de leur famille et à certains marqueurs culturels, dans le but de "faire plaisir" à leur famille ou pour revendiquer une réciprocité, voire être en mesure de négocier des "extras" lorsqu'ils se retrouvent avec leurs pairs en contexte scolaire. 